\section{Expanding pulmonary rehabilitation capacity. One size won't fit all}

\author{
Mike Morgan
}

Pulmonary rehabilitation for people with chronic lung disease is a formal programme of care that includes supervised exercise and self-management training. The results of numerous clinical trials show that it can improve exercise capacity, dyspnoea and health status. These benefits have a larger effect size than can be obtained with any other medical therapy for COPD. The benefits last for approximately 12 months and can be regained with repeat programmes. Effective as it has been in a research setting, the challenge for the pulmonary rehabilitation community is to demonstrate that these positive findings can be reproduced in real life and that there is sufficient capacity in the health system to accommodate all those people that could benefit.

Earlier this year the Royal College of Physicians of London (RCP) produced the second of two publications from the largest audit of pulmonary rehabilitation in England and Wales that has ever been conducted. $^{1}{ }^{2}$ This audit included 7413 patients from 224 programmes in a wide variety of settings and described both the provision and the outcomes of rehabilitation. Even though the headline numbers appear impressive, the authors estimate that given 1.2 million people have COPD in the UK, only about $15 \%$ of patients with significant disability are ever referred for rehabilitation. The reasons for this are not clear but could relate to perceived lack of efficacy by referrers or lack of local capacity. The former is difficult to accept because the benefits of rehabilitation are clearly stated in all evidencebased clinical guidelines for COPD but referral may not yet be second nature to all primary or secondary care clinicians. Insufficient capacity, however, is certainly an important issue. In the RCP audit the median capacity of programmes was between 200 and 300 patients per year which is unlikely to satisfy the country's needs. Ironically, even in some areas where capacity is available, the referrals may not be made because of lack of incentives. The balance of need, supply and

Correspondence to Professor Mike Morgan, Department of Respiratory Medicine, University Hospitals of Leicester NHS Trust, Glenfield Hospital, Leicester, UK; mike.morgan@uhl-tr.nhs.uk demand is not yet in equilibrium. The UK is not the only country where supply is inadequate. A recent global survey of pulmonary rehabilitation providers has also shown that most centres are capable of processing only relatively small numbers of patients. ${ }^{3}$ The RCP audit also examined the outcomes of patients who underwent the programmes. At first sight the results may have seemed a little disappointing with only $42 \%$ of patients referred for rehabilitation gaining measureable benefit. However, these headline figures mask an incremental attrition. Only just over two-thirds of patients will turn up to an assessment after referral suggesting that rehabilitation is not sold strongly enough. A further smaller proportion will not want to continue after the process is explained to them at assessment. Once established on the programme $71 \%$ of patients manage to see it through to completion. Those patients that do eventually complete the programme get similar benefits to those seen in research trials with $78 \%$ obtaining a minimally important clinical difference in an exercise or health status outcome. It is hugely reassuring to see that such excellent results can be obtained in a variety of settings across the country. The challenge however, is to expand capacity for pulmonary rehabilitation and to make it attractive to referrers and patients alike while maintaining sufficient quality and outcome.

The setting for pulmonary rehabilitation may vary. The audit uncovered directly supervised programmes that functioned in a variety of settings from acute hospitals to community centres and village halls. We have learnt in the past that to achieve the best outcomes the setting is less important than the skill and commitment of the staff that deliver the programme. ${ }^{4}$ In spite of these varied settings it is likely that we will still fall short of providing capacity for all those who could benefit. For this reason, innovation is required to search for home-based or digitally delivered solutions than can provide an alternative solution. There are some obvious candidates including telemedicine solutions for remote locations and manual or internet-based selfinstruction. One alternative is to provide rehabilitation in the home setting with a degree of supervision provided by visiting staff or telephone contact. In theory, this approach could be more acceptable to patients who have difficulty accessing the traditional outpatient programme and could also have advantages by incorporating the principles of rehabilitation and behaviour change into the relevance of daily life. Several studies have explored pulmonary rehabilitation in the patient's own home in the past with variable results. One early study showed that patients with severe dyspnoea offered personalised rehabilitation in the home did not do as well as those who attended a centre. ${ }^{5}$ However, in this case the training intensities and modalities were not identical. A more recent well conducted study in Canada demonstrated equivalent results for home-based rehabilitation compared with traditional centre-based programmes. ${ }^{6}$ The benefit in health status was similar after 1 year. In this case the patients attended the hospital initially for a 4-week educational programme and those randomised subsequently to homebased rehabilitation were issued with equivalent exercise equipment. While the physical benefits appeared similar in this hybrid format there may be no gain in overall health economic benefit unless the home programme delivers better value.

Thorax contains a well conducted randomised trial of low cost, home-based rehabilitation compared with the conventional hospital outpatient-based programme. ${ }^{7}$ The paper by Anne Holland and colleagues from Melbourne describes a randomised trial of home-based versus standard centre-based rehabilitation. In this case the home-based service contained the familiar components but was initiated in the patient's home and then supervised by weekly structured telephone calls designed to encourage and maintain motivation and adherence. The primary outcome was the 6 min walk distance and the study was powered to demonstrate equivalence on an intention-to-treat analysis. A future paper may describe the health economic comparison. The overall results suggest that home-based rehabilitation is equivalent to the centre-based service. Although this appears a reasonable conclusion from the data, it is revealing to look behind headlines. Unusually, the centre-based results show much poorer improvements in 6 min walk distance than would be expected from per protocol analysis of other studies or the real life experience from the RCP audit. On closer observation, there appears to be a much higher dropout rate after enrolment in the hospital arm compared with the home- 
based rehabilitation (51\% vs 9\%) which might account for the weaker outcome. Indeed the authors also provide a per protocol analysis which shows more respectable results for the hospital programme. Another point to note is that $42 \%$ of the eligible patients who were excluded from randomisation did so because they actively wished to join the hospital programme. This paper raises a number of important issues. It probably does not have the final word as to whether home-based rehabilitation is equivalent to or even superior in some ways to centre-based rehabilitation. The evident issues around willingness to be randomised and the higher-than-average dropout rate in one arm may have taken the gloss off the study. Perhaps such questions cannot be answered in a single-centre trial with randomisation but require a comparison of similar centres that have evolved different types of programmes. Standardisation of content and delivery and subsequent audit and health economic assessment might help in this respect.

It is clear that effective supervised pulmonary rehabilitation can be performed in a number of different settings. The choice of setting may depend on what is locally commissioned or delivered by the provider. The local geography and access routes will also be an important consideration. One possible inference from the Holland study is that patients demonstrate a preference and that this might have an influence on the outcome of a therapy that they have to take part in with enthusiasm and commitment to obtain the optimum benefit. It follows therefore that, where possible, patients should be offered a choice of venue to maximise adherence. Given the possible plurality of future provision it will be important to ensure that formal rehabilitation programmes do provide equivalent outcomes. In England we have excellent evidence-based clinical guidelines and quality standards for pulmonary rehabilitation. ${ }^{8} 9$ We are also fortunate have an ongoing national audit to identify variation. Given the variety of possible providers it is important for commissioners and service providers to demonstrate that the health professionals that deliver a programme are competent to do so by meeting predefined quality standards. Pulmonary rehabilitation is an excellent subject for clinical service accreditation because the area is circumscribed and the outcomes are measured routinely. Such a scheme is currently being developed jointly by the British Thoracic Society and the Royal College of Physicians. Finally, there remains a huge challenge in delivering pulmonary rehabilitation on a large scale to meet the potential demand. It may be worth considering whether everybody with a degree of disability from COPD requires a fullformat supervised pulmonary rehabilitation programme. There are alternatives including mindfulness programmes, health coaching, local exercise programmes and internet applications that have some benefit. ${ }^{10-12}$ While these may not produce the same durable outcomes as a formal programme, they may have sufficient benefit to allow patients to function until they need a formal programme. Such a graded approach may slow down the impact of impending disability and also precondition people to understanding the benefits of a formal pulmonary rehabilitation programme when that becomes necessary. The pulmonary rehabilitation field has come a long way in the last three decades. We have begun to understand what works but in future we will need to deliver physical activity and self-management schemes at scale and in a format that suits the individual. Pulmonary rehabilitation services should emulate other therapeutic advances and address the issue of personalised delivery and not continue with the outdated mantra that once size fits all.

\section{Competing interests None declared.}

Provenance and peer review Commissioned; externally peer reviewed.

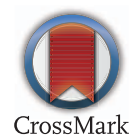

To cite Morgan M. Thorax 2017;72:4-5.

Published Online First 2 November 2016

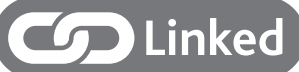

http://dx.doi.org/10.1136/thoraxjnl-2016-208514
Thorax 2017;72:4-5.

doi:10.1136/thoraxjnl-2016-209345

\section{REFERENCES}

1 Steiner M, Holzhauer-Barrie J, Lowe D, et al. Pulmonary Rehabilitation: Steps to breathe better. National Chronic Obstructive Pulmonary Disease (COPD) Audit Programme: Clinical audit of Pulmonary Rehabilitation services in England and Wales 2015. National clinical audit report. London: RCP, Feb 2016.

2 Steiner M, Holzhauer-Barrie J, Lowe D, et al. Pulmonary Rehabilitation: Time to breathe better. National Chronic Obstructive Pulmonary Disease (COPD) Audit Programme: Resources and organisation of Pulmonary Rehabilitation services in England and Wales 2015. National organisational audit report. London: RCP, Nov 2015.

3 Spruit MA, Pitta F, Garvey C, et al. Differences in content and organisational aspects of pulmonary rehabilitation programmes. Eur Respir J 2014;43:1326-37.

4 Waterhouse JC, Walters SJ, Oluboyede Y, et al. A randomised $2 \times 2$ trial of community versus hospital pulmonary rehabilitation, followed by telephone or conventional follow-up. Health Technol Assess 2010;14:i-v, vii-xi, 1-140.

5 Wedzicha JA, Bestall JC, Garrod R, et al. Randomized controlled trial of pulmonary rehabilitation in severe chronic obstructive pulmonary disease patients, stratified with the MRC dyspnoea scale. Eur Respir J 1998;12:363-9.

6 Maltais F, Bourbeau J, Shapiro S, et al. Effects of home-based pulmonary rehabilitation in patients with chronic obstructive pulmonary disease: a randomized trial. Ann Intern Med 2008;149: 869-78.

7 Holland AE, Mahal A, Hill CJ, et al. Home-based rehabilitation for chronic obstructive pulmonary disease using minimal resources. Thorax 2017;72: 57-65.

8 Bolton CE, Bevan-Smith EF, Blakey JD, et al. British Thoracic Society guideline on pulmonary rehabilitation in adults. Thorax 2013;68(Suppl 2): ii1-30.

9 British Thoracic Society. Quality Standards for Pulmonary Rehabilitation in Adults. British Thoracic Society Reports 2014;6. May 2014. https://www.britthoracic.org.uk/document-library/dinical-information/ pulmonary-rehabilitation/bts-quality-standards-forpulmonary-rehabilitation-in-adults (accessed 25 Oct 2016).

10 Benzo R, Vickers K, Novotny PJ, et al. Health coaching and chronic obstructive pulmonary disease rehospitalization. A randomized study. Am I Respir Crit Care Med 2016;194:672-80.

11 Mantoani LC, Rubio N, McKinstry B, et al. Interventions to modify physical activity in patients with COPD: a systematic review. Eur Respir J 2016;48:69-81.

12 Mitchell KE, Johnson-Warrington V, Apps LD, et al. A self-management programme for COPD: a randomised controlled trial. Eur Respir J 2014;44:1538-47. 